\title{
Calorimetric and Computational Study of Indanones
}

M. Agostinha R. Matos, Margarida S. Miranda, Manuel J. S. Monte, Luís M. N. B. F. Santos,

Victor M. F. Morais, James S. Chickos, Patamaporn Umnahanant, Joel F. Liebman

Supporting Information

Table S1. Retention Times for 1-Indanone

\begin{tabular}{lccccccc}
\hline $\mathrm{T} / \mathrm{K}$ & 404.4 & 409.4 & 414.5 & 419.5 & 424.7 & 429.6 & 434.5 \\
\hline & & & & $\mathrm{t} / \mathrm{min}$ & & & \\
$\mathrm{CH}_{2} \mathrm{Cl}_{2}$ & 0.312 & 0.306 & 0.308 & 0.308 & 0.315 & 0.309 & 0.31 \\
4-heptanone & 0.571 & 0.534 & 0.511 & 0.489 & 0.478 & 0.454 & 0.441 \\
acetophenone & 1.227 & 1.097 & 0.999 & 0.914 & 0.854 & 0.781 & 0.729 \\
cyclooctanone & 1.577 & 1.397 & 1.258 & 1.14 & 1.053 & 0.953 & 0.881 \\
6-undecanone & 2.915 & 2.479 & 2.144 & 1.868 & 1.66 & 1.451 & 1.296 \\
1-indanone & 3.294 & 2.827 & 2.463 & 2.16 & 1.93 & 1.694 & 1.518 \\
methyl laurate & 10.525 & 8.523 & 7.001 & 5.803 & 4.89 & 4.073 & 3.459 \\
\hline
\end{tabular}


Table S2. A Summary of the Gas Chromatographic Correlation for 1-Indanone

\begin{tabular}{lccccc}
\hline & intercept & $\begin{array}{c}\Delta_{s h n}{ }^{g} H^{o}{ }_{m}(420 \mathrm{~K}) \\
\mathrm{kJ} \cdot \mathrm{mol}^{-1}\end{array}$ & $\begin{array}{c}\Delta_{l}^{g} H^{o}{ }_{m}(298 \mathrm{~K}) \\
\mathrm{kJ} \cdot \mathrm{mol}^{-1} \\
\text { lit }\end{array}$ & $\begin{array}{c}\Delta_{l}^{g} H^{o}{ }_{m}(298 \mathrm{~K}) \\
\mathrm{kJ} \cdot \mathrm{mol}^{-1} \\
\mathrm{calcd}\end{array}$ \\
\hline 4-heptanone & 3953.1 & 11.131 & 32.865 & $46.7^{\mathrm{a}}$ & 48.0 \\
acetophenone & 4520.2 & 11.273 & 37.579 & $55.4^{\mathrm{b}}$ & 53.5 \\
cyclooctanone & 4606.1 & 11.161 & 38.293 & $54.4^{\mathrm{c}}$ & 54.3 \\
6-undecanone & 5625.4 & 12.961 & 46.767 & $63.5^{\mathrm{d}}$ & 64.1 \\
1-indanone & 5235.0 & 11.859 & 43.522 & & $60.4 \pm 2.8^{\mathrm{e}}$ \\
methyl laurate & 6824.8 & 14.561 & 56.739 & $75.6^{\mathrm{f}}$ & 75.6 \\
\hline
\end{tabular}

${ }^{\mathrm{a}}$ ref [1];

${ }^{\mathrm{b}}$ ref [2];

${ }^{\mathrm{c}}$ ref [3];

d ref [4];

${ }^{\mathrm{e}} \mathrm{a} \Delta_{l}^{g} H^{o}{ }_{m}(333 \mathrm{~K})$ value of $60.3 \mathrm{~kJ} \cdot \mathrm{mol}^{-1}$ has been reported; ${ }^{12}$

${ }^{\mathrm{e}}$ ref [5].

$\Delta_{l}^{g} H_{m}^{o}(298.15 \mathrm{~K}) / \mathrm{kJ} \cdot \mathrm{mol}^{-1}=(1.156 \pm 0.074) \Delta_{s l n}^{g} H_{m}(402)+(10.05 \pm 1.39),\left(\mathrm{r}^{2}=0.9879\right)$ 
Table S3. Retention Times for 2-Indanone

\begin{tabular}{lccccccc}
\hline $\mathrm{T} / \mathrm{K}$ & 404 & 409.1 & 414.1 & 419.1 & 424.1 & 429.1 & 434.1 \\
\hline $\mathrm{CH}_{2} \mathrm{Cl}_{2}$ & 0.398 & 0.399 & 0.394 & 0.391 & 0.395 & 0.397 & 0.397 \\
4-heptanone & 0.716 & 0.678 & 0.645 & 0.615 & 0.595 & 0.576 & 0.558 \\
acetophenone & 1.536 & 1.384 & 1.259 & 1.15 & 1.063 & 0.987 & 0.921 \\
cyclooctanone & 1.978 & 1.764 & 1.587 & 1.435 & 1.312 & 1.205 & 1.113 \\
2-indanone & 3.158 & 2.752 & 2.419 & 2.14 & 1.911 & 1.716 & 1.552 \\
6-undecanone & 3.651 & 3.125 & 2.703 & 2.353 & 2.069 & 1.833 & 1.636 \\
methyl laurate & 13.185 & 10.729 & 8.828 & 7.31 & 6.106 & 5.138 & 4.363 \\
\hline
\end{tabular}


Table S4. A Summary of the Gas Chromatographic Correlation for 2-Indanone

\begin{tabular}{lccccc}
\hline & -slope & intercept & $\begin{array}{c}\Delta_{s h n}{ }^{g} H^{o}{ }_{m}(419 \mathrm{~K}) \\
\mathrm{kJ} \cdot \mathrm{mol}^{-1}\end{array}$ & $\begin{array}{c}\Delta_{l}^{g} H^{o}{ }_{m}(298 \mathrm{~K}) \\
\mathrm{kJ} \cdot \mathrm{mol}^{-1} \\
\text { lit }\end{array}$ & $\begin{array}{c}\Delta_{l}^{g} H^{o}{ }_{m}(298 \mathrm{~K}) \\
\mathrm{kJ} \cdot \mathrm{mol}^{-1} \\
\text { calcd }\end{array}$ \\
\hline 4-heptanone & 3946.1 & 10.916 & 32806 & $46.7^{\mathrm{a}}$ & 48.0 \\
acetophenone & 4513.6 & 11.047 & 37524 & $55.4^{\mathrm{b}}$ & 53.5 \\
cyclooctanone & 4609 & 10.955 & 38317 & $54.4^{\mathrm{c}}$ & 54.4 \\
2-indanone & 5076.9 & 11.555 & 42207 & & $58.9 \pm 2.8$ \\
6-undecanone & 5625.7 & 12.749 & 46769 & $63.5^{\mathrm{d}}$ & 64.1 \\
methyl laurate & 6826.2 & 14.353 & 56750 & $75.6^{\mathrm{e}}$ & 75.6 \\
\hline
\end{tabular}

${ }^{\mathrm{a}}$ ref [1];

${ }^{\mathrm{b}}$ ref [2];

${ }^{c}$ ref [3];

${ }^{\mathrm{d}}$ ref [4];

${ }^{\mathrm{e}}$ ref [5].

$\Delta_{l}^{g} H^{o}{ }_{m}(298.15 \mathrm{~K}) / \mathrm{kJ} \cdot \mathrm{mol}^{-1}=(1.153 \pm 0.074) \Delta_{s l n}^{g} H_{m}(419)+(10.194 \pm 1.40),\left(\mathrm{r}^{2}=0.9878\right)$ 
Table S5. Retention Times for 1,3-Indanedione

\begin{tabular}{lccccccc}
\hline $\mathrm{T} / \mathrm{K}$ & 439.3 & 444.2 & 449.2 & 454.1 & 459.1 & 464.0 & 473.9 \\
\hline & & & \multicolumn{5}{c}{$\mathrm{t} / \mathrm{min}$} \\
$\mathrm{CH}_{2} \mathrm{Cl}_{2}$ & 0.505 & 0.506 & 0.51 & 0.515 & 0.519 & 0.525 & 0.518 \\
1,4-cyclohexanedione & 1.127 & 1.064 & 1.009 & 0.965 & 0.925 & 0.905 & 0.819 \\
1,3-indanedione & 2.835 & 2.545 & 2.297 & 2.09 & 1.916 & 1.813 & 1.489 \\
1,4-naphthoquinone & 3.563 & 3.172 & 2.839 & 2.561 & 2.327 & 2.185 & 1.762 \\
Benzil & 16.535 & 13.905 & 11.761 & 10.02 & 8.61 & 7.638 & 5.53 \\
\hline
\end{tabular}


Table S6. A Summary of the Gas Chromatographic Correlation for 1,3-Indanedione

\begin{tabular}{lccccc}
\hline & & & $\Delta_{s l n}{ }^{g} H^{o}{ }_{m}(419 \mathrm{~K})$ & $\Delta_{l}^{g} H^{o}{ }_{m}(298 \mathrm{~K})$ \\
& -slope & intercept & $\mathrm{kJ} \cdot \mathrm{mol}_{l}{ }^{g} H^{o}{ }_{m}(298 \mathrm{~K})$ & $\begin{array}{c}\mathrm{kJ} \cdot \mathrm{mol}^{-1} \\
\text { lit }\end{array}$ & $\begin{array}{c}\mathrm{kJ} \cdot \mathrm{mol}^{-1} \\
\text { calcd }\end{array}$ \\
\hline 1,4-cyclohexanedione & 4282.4 & 10.225 & 35602 & $68.7^{\mathrm{a}}$ & 68.7 \\
1,3-indanedione & 5151.6 & 10.884 & 42828 & & $72.6 \pm 2.0$ \\
1,4-naphthoquinone & 5297.9 & 10.945 & 44045 & $73.2^{\mathrm{b}}$ & 73.3 \\
Benzil & 6896.8 & 12.93 & 57337 & $80.6^{\mathrm{b}}$ & 80.6 \\
\hline
\end{tabular}

${ }^{\mathrm{a}} \operatorname{Ref}[6,7]$

${ }^{\mathrm{b}}$ see Table 7.

$\Delta_{l}^{g} H_{m}^{o}(298.15 \mathrm{~K}) / \mathrm{kJ} \cdot \mathrm{mol}^{-1}=(0.548 \pm 0.006) \Delta_{s l n}^{g} H_{m}(419)+(49.13 \pm 1.0),\left(\mathrm{r}^{2}=0.9999\right)$ 
Table S7. Calculation of Vaporization Enthalpies from Sublimation and Fusion Enthalpies

\begin{tabular}{lcccccc}
\hline & $\begin{array}{c}\Delta_{c r}{ }^{g} H^{o}{ }_{m}(298 \mathrm{~K}) \\
\mathrm{kJ} \cdot \mathrm{mol}^{-1} \\
\text { lit }\end{array}$ & $\begin{array}{c}C_{p l} / C_{p c r} \\
\mathrm{~J} \mathrm{~mol}^{-1} \cdot \mathrm{K}^{-1}\end{array}$ & $\begin{array}{c}\Delta_{c r}{ }^{l} H^{o}{ }_{m}\left(T_{\text {fus }}\right) \\
\mathrm{kJ} \cdot \mathrm{mol}^{-1}\end{array}$ & $\begin{array}{c}T_{\text {fus }} / \mathrm{K} \Delta_{c r}{ }^{l} H^{o}{ }_{m}(298 \mathrm{~K}) \\
\mathrm{kJ} \cdot \mathrm{mol}^{-1} \\
\mathrm{calcd}\end{array}$ & $\begin{array}{c}\Delta_{l}^{g} H^{o}{ }_{m}(298 \mathrm{~K}) \\
\mathrm{kJ} \cdot \mathrm{mol}^{-1}\end{array}$ \\
\hline Benzil & $99.7^{\mathrm{a}, \mathrm{b}}$ & $351.6 / 248$ & $23.56^{\mathrm{c}}$ & 368 & $19.1^{\mathrm{d}}$ & $80.6^{\mathrm{e}}$ \\
1,4-naphthoquinone & $91.2^{\mathrm{f}}$ & $254.2 / 187.4$ & $22.8^{\mathrm{g}}$ & 397 & $18.0^{\mathrm{d}}$ & $73.2^{\mathrm{e}}$
\end{tabular}

Adjusted to $T=298.15 \mathrm{~K}$ using the following equation: $\Delta_{c r}{ }^{g} H^{o}{ }_{m}(298 \mathrm{~K}) / \mathrm{kJ}^{\prime} \mathrm{mol}^{-1}=\Delta_{c r}{ }^{g} H_{m}^{o}\left(T_{\mathrm{m}}\right)$ $+\left(0.75+0.15 \mathrm{C}_{\mathrm{pcr}}\right)\left(T_{\mathrm{m}}-298.15 \mathrm{~K}\right) / 1000 \operatorname{ref}[9]$;

${ }^{\mathrm{b}} T_{\mathrm{m}}=329 \mathrm{~K}$, ref [8];

${ }^{c}$ ref [10];

${ }^{\mathrm{d}} \Delta_{\mathrm{cr}}{ }^{1} H_{m}^{\mathrm{o}}(298.15 \mathrm{~K}) / \mathrm{kJ} \cdot \mathrm{mol}^{-1}=\Delta_{\mathrm{cr}}{ }^{1} H_{m}^{\mathrm{o}}\left(T_{f u s}\right)+\left(0.15 C_{p c r}-0.26 C_{p l}-9.83\right)\left(T_{m}-298.15\right) / 1000 ;$ ref [6];

${ }^{\mathrm{e}} \Delta_{l}^{g} H^{o}{ }_{m}(298 \mathrm{~K})=\Delta_{c r}{ }^{g} H^{o}{ }_{m}(298 \mathrm{~K})-\Delta_{c r}{ }^{l} H^{o}{ }_{m}(298 \mathrm{~K})$

${ }^{\mathrm{f}} \mathrm{ref}[11]$

${ }^{\mathrm{g}}$ this work, average of three measurements: $(22.8 \pm 0.7) \mathrm{kJ} \cdot \mathrm{mol}^{-1}$; 


\section{Experimental}

Most substances were purchased from the Aldrich Chemical Company and were used without any further purification. Each was analyzed by gas chromatography and found to be at least 98 mole percent pure. Correlation gas chromatography experiments were performed on an HP 5890 Gas Chromatograph equipped with a split/splitless capillary injection port and a flame ionization detector run at a split ratio of approximately 100/1. Retention times were recorded to three significant figures following the decimal point using HP software interfaced to a computer. The instrument was run isothermally using a $15 \mathrm{~m}$ SPB-5 capillary column. Helium was used as the carrier gas. At the temperatures of the experiments, the solvent, $\mathrm{CH}_{2} \mathrm{Cl}_{2}$ or cyclopentane, is not retained. The retention times of the solvent were used to determine the dead volume of the column. Adjusted retention times, $\mathrm{t}_{\mathrm{a}}$, were calculated by subtracting the measured retention time of the solvent from the retention time of each analyte as a function of temperature usually over a 25 - $30 \mathrm{~K}$ range. Column temperatures were controlled by the gas chromatograph and were monitored independently by using a Fluke $51 \mathrm{~K} / \mathrm{J}$ thermometer. Temperature was maintained constant by the gas chromatograph to $\pm 0.1 \mathrm{~K}$. Duplicate analyses were performed for each to confirm reproducibility. Data for only one run is reported.

Fusion enthalpies were measure on a Perkin Elmer DSC-7 under a nitrogen purge in hermetically sealed aluminum cells at a scan rate of $5 \mathrm{~K} \mathrm{~min}^{-1}$. The fusion temperature reported is an onset temperature. All fusion enthalpy determinations were performed in triplicate. Calibration of the instrument was achieved by using indium as a standard and checked with benzoic acid. 
Table S8. Individual Values of the Massic Energy of Combustion, at $T=298.15 \mathrm{~K}$.

\begin{tabular}{ccc}
\hline \multicolumn{3}{c}{$-\Delta_{\mathrm{c}} u^{\circ} /\left(\mathrm{J} \mathrm{g}^{-1}\right)$} \\
\hline 1-indanone & 2-indanone & 1,3 -indanedione \\
\hline 34381.91 & 34430.16 & 28339.88 \\
34348.27 & 34442.49 & 28337.32 \\
34343.54 & 34403.30 & 28310.48 \\
34324.40 & 34397.16 & 28299.51 \\
34333.07 & 34392.70 & 28307.85 \\
34331.82 & 34419.57 & 28297.95 \\
34360.35 & & 28317.66 \\
34345.22 & & 28299.21 \\
& $-<\Delta_{\mathrm{c}} u^{\circ}>\left(\mathrm{J} \mathrm{g}^{-1}\right)$ \\
$34346.1 \pm 6.5$ & $34414.2 \pm 8.1$ & $28313.7 \pm 5.9$ \\
\hline
\end{tabular}


Table S9. Most Relevant B3LYP/6-311G** Geometrical Parameters of the

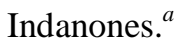

\begin{tabular}{|c|c|c|c|c|c|}
\hline & \multicolumn{2}{|c|}{ 1-indanone } & \multirow[t]{2}{*}{ 2-indanone } & \multicolumn{2}{|c|}{ 1,3-indanedione } \\
\hline & calc & $\exp ^{1}$ & & calc & $\exp ^{34}$ \\
\hline $\mathrm{R}_{1,2}$ & 1.537 & 1.524 & 1.537 & 1.531 & 1.507 \\
\hline $\mathrm{R}_{2,3}$ & 1.546 & 1.536 & 1.537 & 1.532 & 1.516 \\
\hline $\mathrm{R}_{3,4}$ & 1.517 & 1.516 & 1.507 & 1.491 & 1.477 \\
\hline $\mathrm{R}_{4,5}$ & 1.395 & 1.393 & 1.402 & 1.397 & 1.387 \\
\hline $\mathrm{R}_{1,5}$ & 1.483 & 1.478 & 1.507 & 1.492 & 1.478 \\
\hline $\mathrm{R}_{1,6}$ & 1.209 & 1.217 & & 1.207 & 1.214 \\
\hline $\mathrm{R}_{2,6}$ & & & 1.202 & & \\
\hline $\mathrm{R}_{3,7}$ & & & & 1.207 & 1.211 \\
\hline$\Theta_{1,2,3}$ & 106.4 & 106.2 & 108.8 & 105.3 & 104.3 \\
\hline$\Theta_{2,3,4}$ & 104.6 & 104.7 & 104.2 & 107.0 & 107.8 \\
\hline$\Theta_{3,4,5}$ & 111.6 & 111.4 & 111.4 & 110.3 & 109.9 \\
\hline$\Theta_{4,5,1}$ & 110.3 & 109.9 & 111.4 & 110.4 & 109.6 \\
\hline$\Theta_{5,1,2}$ & 107.1 & 107.5 & 104.2 & 107.0 & 108.2 \\
\hline$\Theta_{6,1,2}$ & 125.9 & 125.9 & & 126.7 & 125.9 \\
\hline$\Theta_{6,2,3}$ & & & 125.6 & & \\
\hline$\Theta_{2,3,7}$ & & & & 126.7 & 125.5 \\
\hline$\Theta_{1,2,3,4}$ & 0.2 & 5.4 & 2.7 & 0.0 & 2.9 \\
\hline$\Theta_{1,5,4,3}$ & 0.0 & -0.4 & 0.2 & 0.1 & 1.0 \\
\hline
\end{tabular}

${ }^{a}$ bond lengths in $\AA$ and bond angles in degrees 
Table S10. Most Relevant B3LYP/6-311G** Geometrical Parameters of the Cyclopentanones. ${ }^{a}$

\begin{tabular}{cccc}
\hline & cyclopentanone & 2-cyclopentenone & 3-cyclopentenone \\
\hline $\mathbf{R}_{1,2}$ & 1.533 & 1.486 & 1.537 \\
$\mathrm{R}_{2,3}$ & 1.535 & 1.337 & 1.505 \\
$\mathrm{R}_{3,4}$ & 1.546 & 1.510 & 1.334 \\
$\mathrm{R}_{4,5}$ & 1.536 & 1.540 & 1.505 \\
$\mathbf{R}_{1,5}$ & 1.534 & 1.538 & 1.537 \\
$\mathrm{R}_{1,6}$ & 1.204 & 1.209 & 1.202 \\
$\Theta_{1,2,3}$ & 104.9 & 110.4 & 103.4 \\
$\Theta_{2,3,4}$ & 104.0 & 113.3 & 112.5 \\
$\Theta_{3,4,5}$ & 104.2 & 104.0 & 112.6 \\
$\Theta_{4,5,1}$ & 105.1 & 105.5 & 103.4 \\
$\Theta_{5,1,2}$ & 108.3 & 106.8 & 108.1 \\
$\Theta_{6,1,2}$ & 125.9 & 127.2 & 126.0 \\
$\Theta_{1,2,3,4}$ & -30.9 & 0.0 & 0.1 \\
$\Theta_{6,1,2,3}$ & -166.9 & 179.9 & 179.7 \\
$\Theta_{1,5,4,3}$ & -29.2 & 0.0 & -0.2 \\
\hline
\end{tabular}

${ }^{a}$ bond lengths in $\AA$ and bond angles in degrees 
Table S11. Calculated Electronic Energies (Hartree), Zero-point Vibrational Energies and Thermal Corrections at $\left.T=298.15 \mathrm{~K}_{(\mathrm{kJ} \mathrm{mol}}{ }^{-1}\right)$.

\begin{tabular}{lcccccc}
\hline Compound & $E_{\mathrm{B} 3 \mathrm{LYP} /}$ & $E_{\mathrm{B} 3 \mathrm{LY} /}$ & $E_{\mathrm{MPW} 1 \mathrm{~B} 95 /}$ & $E_{\mathrm{MPW} 1 \mathrm{~B} 95 /}$ & $E_{\mathrm{ZP} / 6-31 \mathrm{G}^{*}}$ & ${ }^{a} E_{\mathrm{total} / 6-31 \mathrm{G}^{*}}$ \\
& & & & & & \\
\hline 1-indanone & $-423.111 \mathrm{G}^{* *}$ & $\mathrm{cc-pVTZ}$ & $6-311 \mathrm{G}^{* *}$ & ${ }^{\mathrm{cc}-\mathrm{pVTZ}}$ & & \\
2-indanone & -423.112906 & -423.157695 & -422.906916 & -422.946937 & 380.80 & 400.71 \\
1,3-indanedione & -497.153533 & -497.205346 & -496.933278 & -496.980734 & 332.81 & 354.11 \\
indane & -349.078176 & -349.115134 & -348.885566 & -348.917740 & 433.25 & 451.24 \\
cyclopentane & -196.613422 & -196.632608 & -196.480834 & -196.497518 & 371.33 & 384.60 \\
cyclopentanone & -270.650705 & -270.677947 & -270.505464 & -270.530184 & 319.81 & 334.41 \\
2-cyclopentenone & -269.425762 & -269.454083 & -269.289671 & -269.315641 & 258.10 & 271.68 \\
3-cyclopentenone & -269.417927 & -269.446185 & -269.282210 & -269.308010 & 255.88 & 269.42 \\
\hline
\end{tabular}

${ }^{a} E_{\text {total }}(T=298.15 \mathrm{~K})=E_{\text {trans }}+E_{\text {rot }}+E_{\mathrm{ZP}}+\Delta_{0 \mathrm{~K}}^{298.15 \mathrm{~K}} E_{\mathrm{vib}}$ 

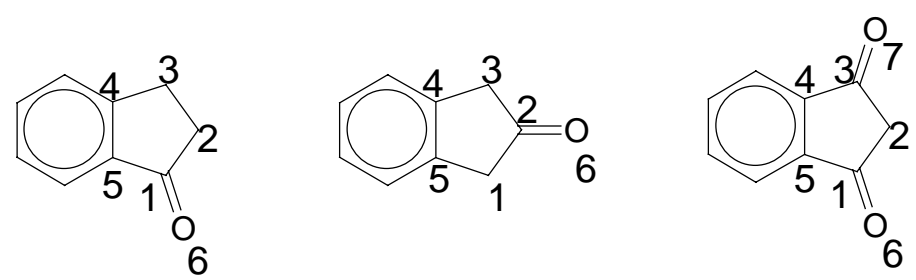

Figure 1. Atom numbering scheme of 1-indanone, 2-indanone and 1,3-indanedione. 


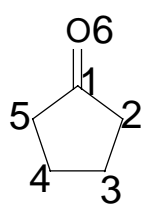

Figure 2. Atom numbering scheme of cyclopentanone, 2-cyclopentenone and 3-cyclopentenone. 
References

1. Ambrose, D.; Ellender, J. H.; Lees, E. B.; Sprake, C. H. S.; Townsend, R. J. Chem. Thermodyn. 1975, 7, 453.

2. Steele, W. V.; Chirico, R. D.; Knipmeyer, S. E.; Nguyen, A.; J. Chem. Eng. Data 1996, 41,1255 .

3. Chickos, J. S.; Hosseini, S.; Hesse, D. G. Thermochim. Acta 1995, 249, 41.

4. Saluja, P. P. S.; Peacock, L. A.; Fuchs, R. J. Am. Chem. Soc. 1979, 101, 1958.

5. Chickos, J. S.; Zhao, H.; Nichols, G. Thermochim. Acta 2004, 424, 111.

6. Umnahanant, P.; Chickos, J. S. J. Chem. Eng. Data 2005, 50, 1720.

7. De Wit, H. G. M.; van Miltenburg, J. C.; De Kruif, C. G. J. Chem. Thermodyn. 1983, 15, 651.

8. Aihara, A. Bull Chem. Soc. Jpn. 1959, 32, 1242.

9. Chickos, J. S.; Hosseini, S.; Hesse, D.G.; Liebman, J. F. Struct. Chem. 1993, 4,271;

Chickos, J. S.; Hesse, D. G.; Liebman, J. F. Struct. Chem. 1993, 4, 261.

10. Andon, R. J. L.; Connett, J. E. Thermochim. Acta 1980, 42, 241.

11. DeKruif, C. G.; Smit, E. J.; Gover, H. A. J. Chem. Phys. 1981, 74, 5838; Ribeiro Da Silva, M. A. V.; Ribeiro Da Silva, M. D. M. C; Taixeira, J. A. S.; Bruce, J. M.; Guyan, P. M.; Pilcher, G. J. Chem. Thermodyn. 1989, 21, 265.

12. Verevkin S. P. Thermochim. Acta 1998, 310, 229. 